\title{
Régénération
}

\section{de l'iodomercurate de potassium usagé pour favoriser son utilisation continue en coproscopie}

\author{
par J.-P. RAYNAUD et G. BRUNAULT \\ (Collaboration technique : J.-M. LÉonhart et F. Gansuana) \\ Station de Recherche et Développement vétérinaire, \\ Pfizer International, B.P. 42, F 37400 Amboise
}

\section{Résumé.}

La technique de coproscopie quantitative que nous avons présentée en 1969 et détaillée en 1970 présente les qualités requises pour un laboratoire de recherche ou de diagnostic. Mais l'utilisation d'Iodomercurate de Potassium comme solution dense, à raison de $70 \mathrm{ml}$ par coproscopie, entraîne des dépenses élevées. Nous proposons un procédé de régénération complète (à $95 \%$ ) de l'Iodomercurate usagé : précipitation par la chaux et clarification par du noir animal. Avec ce procédé, la technique de coproscopie que nous préconisons est d'un prix de revient négligeable, quelques centimes seulement.

\section{Summary.}

Regeneration of used mercuric and potassium iodide for an economical use in egg-counts routines.

We presented in 1969 and detailed in 1970 a quantitative egg count technique which presents the qualities necessary for a research or diagnosis laboratory. But using mercuric and potassium iodide at $70 \mathrm{ml}$ for each examination is quite expensive. We now propose a method for the complete renewal (at $95 \%$ ) of used mercuric and potassium iodide solution: precipitation with lime and clarification with animal charcoal. With this procedure the egg count technique we propose is at a negligeable operating cost, a few centimes only. 
Nous avons présenté (Raynaud, 1969) une technique de coproscopie quantitative dérivée de Mac Master modifiée par Gordon et Whitlock, 1939.

Nous avons pu démontrer (Raynaud, 1970) sur des bases expérimentales précises et des résultats chiffrés que cette technique est :

- polyvalente (elle détecte toutes les formes parasitaires éliminées: Cestodes, Trématodes, Nématodes, œufs ou larves) ;

- simple (un seul prélèvement de $5 \mathrm{~g}$ de matières fécales est manipulé) ;

- rapide et relativement peu onéreuse (le liquide cher, l'iodomercurate de potassium est partiellement régénéré et récupéré) ;

- précise, ce que permet l'utilisation de la lame de Mac Master;

- enfin, sensible, car le seuil de détection apporté par la lame de Mac Master est amélioré par l'examen complémentaire d'une lame de flottaison. Deux éléments négatifs persistent: les æuufs à coque mince (Fasciola) sont déformés, la manipulation doit se faire avec quelques précautions (gants chirurgicaux et protection des yeux).

Cette technique peut être caractérisée comme une coproscopie quantitative en lame de Mac Master dont le coefficient de multiplication peut être de 15. Ce seuil est abaissé par l'examen complémentaire d'une lame de flottaison qui apporte une information qualitative.

\section{I. - Intérêt présenté par l'iodomercurate de potassium donc par sa régénération.}

Nous ne détaillerons pas les avantages de l'Iodomercurate de Potassium de Janesckso et Urbanek, 1931, mais nous devons reconnaître que c'est la seule solution qui :

— du fait de sa densité, permet la flottaison d'œufs petits ou gros et de larves ;

- du fait de propriétés particulières, digère et réduit les débris, ce qui est peut-être la cause du bon «rendement» de cette solution pour toutes les espèces;

Pour les inconvénients, comme nous l'avons publié en 1970 (ceci est connu et est donné par J. Euzéby, 1958), nous retenons :

- la déformation des œufs de Fasciole et l'impossibilité de les différencier des Paramphistomidés ;

- la manipulation avec précautions: la phase essentielle est l'agitation magnétique qui doit se faire derrière un écran protecteur en plexiglass; ceci est réalisé aisément au laboratoire;

- le prix; nous avons travaillé pendant plusieurs années avec une technique de récupération de l'Iodomercurate de Potassium qui en diminuait le prix de moitié. 
C'est donc sur ce point que nous avons essayé d'apporter une amélioration. Nous avions proposé une régénération par le noir animal, mais nous utilisons aujourd'hui sur une idée de J.-M. Leonhart une méthode de récupération de l'Iodomercurate de Potassium qui permet d'utiliser pratiquement sans frais la même solution, régénérée à $90 \%$.

Nous avons ainsi résolu le problème financier difficile pour un laboratoire de diagnostic et de recherche qui utilise un volume approximatif de 2 à 5 litres d'Iodomercurate de Potassium par semaine.

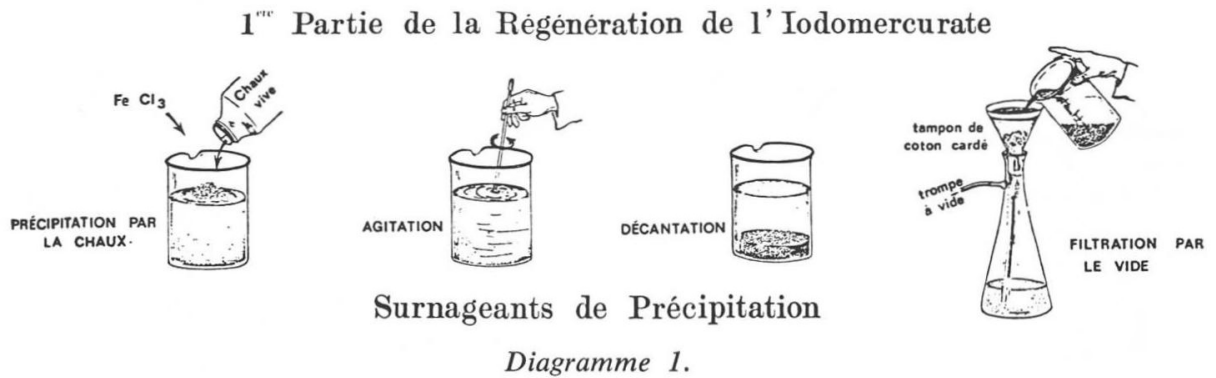

\section{II. - Régénération.}

Les opérations principales sont résumées dans les diagrammes 1,2 et 3 .

\section{a) Matériel à régénérer.}

Nous avons au laboratoire soit les suspensions diluées venant des cellules de Mac Master et des flottaisons, soit mis en pots différents un matériel plus dense, plus chargé, venant des gâteaux de précipitation des premiers et des débris retenus par les tamis. Les deux sources de produits à régénérer sont utilisées séparément.

\section{b) Principe de régénération.}

Pour les suspensions diluées, on fait une défécation par la chaux vive + perchlorure de fer, une clarification complémentaire par du noir animal; les liquides sont réajustés en $\mathrm{pH}$ et densité. Les «gâteaux» de filtration et débris de tamisage sont au contraire dilués en eau, filtrés, clarifiés en noir animal et les liquides réajustés comme précédemment.

c) Précipitation et filtration des suspensions diluées.

1) Précipitation par la chaux.

A 2 litres ou plus d'iodomercurate à régénérer, $30 \mathrm{~g}$ environ de chaux vive en poudre (technique) sont ajoutés et $5 \mathrm{ml}$ environ de perchlorure de fer. 
2) Agitation.

Le mélange se fait avec un agitateur plastique manipulé à la main ; on laisse décanter.

\section{3) Décantation.}

$\mathrm{Au}$ bout d'un quart d'heure, la phase liquide représentant $75 \%$ environ du volume total.

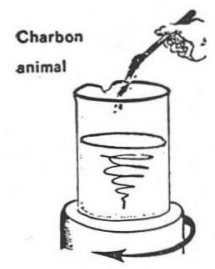

AGITATION MAGNÉTIQUE

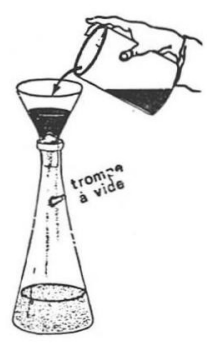

$2^{\text {ème }}$ FILTRATION

PAR LE VIDE

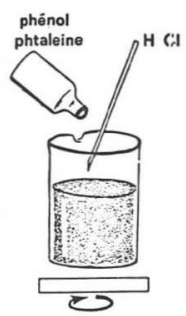

AJUSTEMENT du $\mathrm{Ph}$

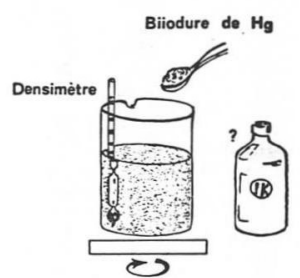

AJUSTEMENT densité

$2^{\text {eme }}$ Partie de la Régénération de l' Iodomercurate

Diagramme 2.

4) $1^{\text {re }}$ FiLtration sous VIDE.

Sur un Erlenmeyer branché sur trompe à eau, on fixe un entonnoir à poudre à large ouverture obturé par un gros bouchon de coton cardé trempé d'eau. On filtre la phase liquide.

d) Phase finale : clarification, ajustement du pH et de la densité.

5) Clarification par du NoIr animal.

La solution filtrée est mise sur un agitateur magnétique; on ajoute $20 \mathrm{~g}$ environ de noir animal ; après l'agitation, on laisse reposer quelques minutes.

6) $2^{e}$ Filtration sous vide.

Avec le même appareillage que pour la $1^{\text {re }}$ filtration. 
7) AJustement du pH.

Avec quelques gouttes de phénolphtaléine on contrôle le $\mathrm{pH}$ qui est à 11,7 environ; on ramène la solution au jaune par $\mathrm{HCl}$ pur; le $\mathrm{pH}$ est alors de 8,2-8,6, ce qui correspond au $\mathrm{pH}$ d'une solution d'Iodomercurate neuf.

8) Ajustement de la densité.

Sous contrôle d'un densimètre, on réajuste la densité à 1,44 en utilisant du Biiodure de mercure et de l'iodure de potassium.
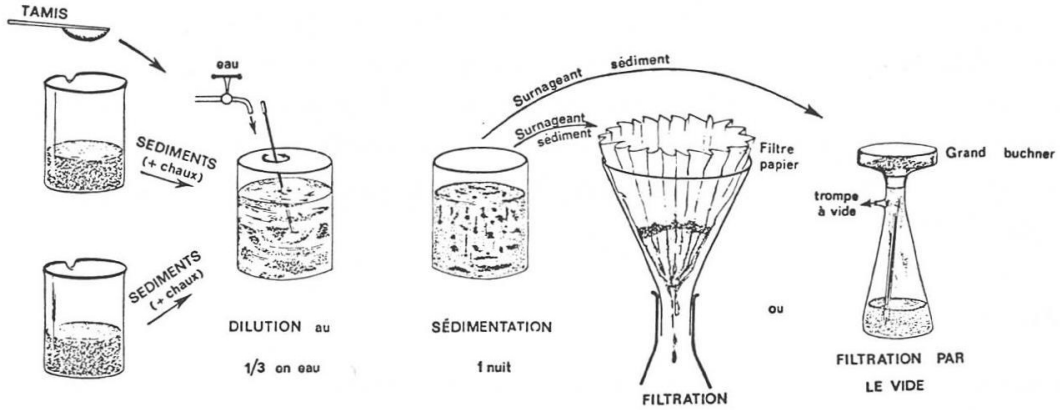

Résidus de Tamisage et Sédiments

Diagramme 3.

e) Dilution et filtration des gâteaux de filtration et débris de tamisage.

Les sédiments issus de la décantation $\left(n^{\circ} 3\right)$ et les résidus de tamisage sont conservés en pots.

1) Dilution des sédiments.

Les sédiments sont dilués par deux fois leur volume en eau.

2) Agitation.

Le mélange se fait à l'agitateur plastique manipulé à la main.

3) SÉdimentation.

On laisse sédimenter une nuit.

4) Filtration.

On filtre le surnageant et une partie du sédiment:

— soit sur papier filtre plissé mis en gros entonnoir. 
- soit sur un grand Büchner de $250 \mathrm{~mm}$ de diamètre au fond duquel on dépose un filtre rond, et mis sur un Erlenmeyer branché sur la trompe à eau.

f) Phase finale : comme la précédente, la technique étant la même en $\mathrm{n}^{\circ} 5$.

\section{III. - Confirmation de l'efficacité.}

Nous avons vérifié précisément:

- que l'on pouvait régénérer un très grand nombre de fois pratiquement le même volume d'Iodomercurate, celui-ci étant mélangé à des matières fécales d'ovins puis régénéré, mélangé à nouveau à d'autres matières d'ovins, puis régénéré, etc... Nous avons choisi les matières d'ovins car elles sont les plus riches en débris végétaux, donc susceptibles d'entraîner des pertes en Iodomercurate plus importantes;

- que les solutions régénérées avaient gardé les mêmes qualités pour la coproscopie quantitative.

CONTRÔLE DU VOLUME RÉGÉNÉRÉ.

Avec des matières d'ovins, donc dans les conditions les plus défavorables, les volumes retrouvés sont contrôlés à chaque régénération.

Protocole: $1^{\text {re }}$ opération: la dilution se fait avec $100 \mathrm{~g}$ de matières fécales $+1400 \mathrm{ml}$ d'Iodomercurate (dans le même rapport que $5 \mathrm{~g}$ de matières $+70 \mathrm{ml}$ de solution dense), on tamise et la suspension est régénérée; elle donne $\mathrm{A} \mathrm{ml}$. Le précipité mélangé aux débris de tamisage donne $\mathrm{B} \mathrm{ml}$.

$2^{\mathrm{e}}$ opération: $\mathrm{A} \mathrm{ml}+\mathrm{B} \mathrm{ml}$ auxquels on ajoute $100(\mathrm{~A}+\mathrm{B}) / 1400 \mathrm{~g}$, soit $\mathrm{A}+\mathrm{B} / 14 \mathrm{~g}$ de matières fécales fraîches. L'opération est répétée 10 fois. Au bout de 10 fois, on retrouve en Iodomercurate régénéré 50 à $80 \%$ du volume initial. On a donc perdu 2 à $5 \%$, soit $5 \%$ au maximum de la solution initiale à chaque opération.

Nous avons vérifié que $65 \%$ de l'Iodomercurate est retrouvé dans les suspensions de matières fécales et $30 \%$ dans les précipités mélangés aux débris de tamisage. Si donc, la régénération est complète suivant la technique que nous indiquons, il y a chez les ovins une perte de $5 \%$ au maximum par coproscopie. Si la régénération, suivant la même technique, ne se faisait que sur les suspensions, la perte serait de $35 \%$ à chaque opération, ce qui est important et justifie la régénération complète.

Autres ESPÈCES.

Pour les Bovins, les rendements sont meilleurs qu'en Ovins, les pertes se situant à un maximum de $3 \%$. Pour les Porcins, Canins et les informations que nous avons en Humains, les pertes sont nulles. 
Dans ces espèces, on peut se contenter de ne régénérer que les suspensions pour une perte de $10 \%$ environ.

COÂT D'UNE COPROSCOPIE.

Chez les Ovins, les pertes s'établissant de 2 à $5 \%$, en ne tenant pas compte de la mise de fonds initiale, la coproscopie, suivant la technique que nous recommandons ( $5 \mathrm{~g}$ de matières fécales $+70 \mathrm{ml}$ d'Iodomercurate), revient à $5 \%$ du prix total de la solution introduite. Pour $4 \mathrm{~F}$ de prix total, le coût d'une coproscopie est donc ramené à $0 F 20$, ce qui est négligeable.

Pour les autres espèces, ce coût est encore inférieur. Cet aspect financier peut paraître superflu, mais nous reprécisons que depuis plusieurs années nous sommes «consommateurs» de 2 à 5 litres d'lodomercurate par semaine.

CONTRôle de L'EFFicacité APRÈS RÉGÉNÉRATION.

Nous avons vérifié que ni le rendement, ni l'homogénéité des résultats, ni la sensibilité en lame de Mac Master ou en flottaison complémentaire n'étaient modifiés sur un même prélèvement portant Fasciola, Dicrocoelium et «Strongles Digestifs » que l'on utilise de l'Iodomercurate frais ou de l'Iodomercurate entièrement régénéré plusieurs fois de suite.

\section{IV. - Conclusion.}

Dans la technique que nous avons publiée en 1970 et que nous pratiquons en routine au laboratoire depuis plus de six ans, c'est un volume de $70 \mathrm{ml}$ d'Iodomercurate de Potassium qui est utilisé à chaque manipulation. Nous «consommons » donc de 2 à 5 litres d'Iodomercurate de Potassium par semaine et il n'est pas nécessaire de détailler les raisons financières qui nous ont poussés à régénérer l'Iodomercurate usé pour pouvoir l'utiliser à nouveau sans avoir perdu les qualités de la solution fraîche.

Nous proposons un procédé de régénération de l'Iodomercurate usagé qui permet, dans les conditions les plus difficiles (matières d'ovins qui contiennent beaucoup de débris importants), de retrouver sans frais plus de $95 \%$ de la solution fraîche mise en œuvre.

Nous avons vérifié que l'on pouvait régénérer un très grand nombre de fois pratiquement le même volume d'Iodomercurate et que les solutions régénérées avaient les mêmes caractéristiques et avaient gardé les mêmes qualités pour la coproscopie quantitative.

L'opération consiste en une précipitation par la chaux, filtration sous vide, clarification par du noir animal, deuxième filtration sous vide, ajustement enfin du $\mathrm{pH}$ et de la densité.

Avec ce procédé de régénération, la technique de coproscopie que nous préconisons est d'un prix de revient négligeable, quelques centimes seulement. 


\section{Bibliographie}

EuzeBy (J.), 1958. — Diagnostic expérimental des helminthoses animales. Vigot Frères, édit., Paris.

Raynaud (J.-P.), 1969. - Techniques et Laboratoire Vétérinaire, Série Parasitologie. I. Le Parasitisme des ruminants. Brochure éditée en juin 1969 par les Laboratoires Pfizer (Paris).

Raynaud (J.-P.), 1970. — Etude de l'efficacité d'une technique de coproscopie quantitative pour le diagnostic de routine et le contrôle des infestations parasitaires des bovins, ovins, équins et porcins. Ann. Parasit. hum. comp., 45, (3), 321-342. 